\title{
Basophils Unchained: A Rare Form of Leukemia
}

\author{
De haes I*, Corveleyn H, Schots R, Depreter B, \\ Demeester S, J ochmans K and De Becker A \\ Department of Clinical Hematology, Universitair of \\ Ziekenhuis Brussel (UZ Brussel), Belgium \\ *Corresponding author: De haes I, Department of \\ Clinical Hematology, Universitair of Ziekenhuis Brussel \\ (UZ Brussel), Laarbeeklaan 101, 1090 Brussels, Belgium
}

Received: September 11, 2021; Accepted: October 07, 2021; Published: October 14, 2021

\section{Abstract}

Primary Chronic Basophilic Leukemia (CBL) is an extremely rare disease and thus knowledge of this disease remains limited. There are no universal diagnostic criteria nor has the underlying pathogenetic mechanism been elucidated.

In this peculiar case, we present a 93-year-old woman with an acute symptomatology of fatigue compounded by a manifest leukocytosis with $43 \%$ basophils in peripheral blood. The bone marrow aspirate also showed basophilia, 56\% specifically. Interestingly, Next Generation Sequencing (NGS) on the bone marrow sample revealed three possible driver mutations, namely in the IDH2, DNMT3A, and BCOR gene. To date, NGS has never been performed on samples of patients with primary CBL. Our findings could be of great value in the search for the pathogenetic mechanism of this rare disease and to help find a successful treatment.

Our patient was palliated with hydroxyurea to lower the white blood cells. Initially, this had a good effect, but unfortunately, she died two months after the diagnosis.

Keywords: Myeloproliferative neoplasms; Chronic basophilic leukemia; Next-generation-sequencing

\section{Abbreviations}

CBL: Chronic Basophilic Leukemia; NGS: Next Generation Sequencing; IDH2: Isocitrate Dehydrogenase 2; DNMT3A: DNA (cytosine-5)-methyltransferase 3A; BCOR: BCL6 Corepressor; WBC: White Blood Cell; MPN: Myeloproliferative Neoplasm; IL6: interleukine-6; CHIP: Clonal Hematopoiesis of Indeterminate Potential

\section{Case Presentation}

A 93-year-old woman presented to the emergency department with an acute onset of fatigue, weakness and abdominal pain. Physical examination showed pallor, cachexia, tachycardia, diffuse abdominal pain without hepatosplenomegaly and oedema of the lower extremities. Neurological examination was normal. A complete blood count revealed thrombocytosis $\left(1245000 / \mathrm{mm}^{3}\right)$ and leukocytosis $\left(40900 / \mathrm{mm}^{3}\right)$, with a normal hemoglobin level. Differential count of the white blood cells showed $32 \%$ neutrophils $\left(13090 / \mathrm{mm}^{3}\right)$, $43 \%$ basophils $\left(17590 / \mathrm{mm}^{3}\right), 13 \%$ eosinophils $\left(5320 / \mathrm{mm}^{3}\right)$, and $2 \%$ lymphocytes $\left(820 / \mathrm{mm}^{3}\right)$. Interestingly, a retrospective view of the lab results revealed a normal blood count with no leukocytosis twenty months before. There were no signs of inflammation; liver and kidney function were in the normal range.

A workup was performed for the marked leukocytosis with eosinophilic and basophilic predominant formula. A peripheral blood sample was tested for the presence of fusion genes have to be cursive: BCR-ABL1, ETV6-PDGFRB, FIP1L1-PDGFRA, PCM1-JAK2 V617F mutation, which were all absent.

Next, a bone marrow aspirate and trephine biopsy were performed. The bone marrow aspirate showed an increased percentage of basophils and eosinophils, $56 \%$ and $8 \%$ of all nucleated cells, respectively, without an increase in immature blasts. The basophilic granulocytes showed a normal immunophenotype, i.e. CD38+, CD45+, CD123+ and CD34- and HLA-DR-. Cytogenetic investigation revealed a normal karyotype, hereby excluding presence of a PCM1-JAK2 fusion protein. The trephine biopsy showed a hypercellular bone marrow (80\%) with marked granulopoiesis and moderately increased megakaryopoiesis. Numerous CD123+, $\mathrm{MPO}+$ and CD34- granulocytes were reported. Based on these characteristics a diagnosis of myeloproliferative neoplasm with basophilic differentiation, defined as Chronic Basophilic Leukemia (CBL) was made.

Additionally, we also performed Next Generation Sequencing (NGS) to screen for mutations in a set of myeloid-related genes ${ }^{*}$ with interpretation according to the Belgian ComPerMed guidelines [1]. Analysis revealed a pathogenic missense (R140Q) mutation in IDH2 (isocitrate dehydrogenase 2), a likely pathogenic (P904A) missense variant in DNMT3A (DNA (cytosine-5)-methyltransferase $3 \mathrm{~A}$ ) and two likely pathogenic frameshift variants in BCOR (BCL6 corepressor).

Given the patient's age and the limited options of treatments for CBL, palliative treatment with hydroxyurea (500 mg once daily) was started. With this treatment, the number of platelets and the WBC count dropped, although not enough to be in their respective normal ranges. The patient was then able to be discharged from the hospital. Unfortunately, she died two months after the diagnosis of CBL was made.

\section{Discussion}

Primary chronic basophilic leukemia is an extremely rare Myeloproliferative Neoplasm (MPN) with a limited number of documented cases. Kyle and Pease are often credited as the first to 
have made a clinical description of basophilic leukemia in 1966 [2]. In the last two decades, only six cases were reported [3-6]. In addition to these, there are five cases reported as secondary chronic basophilic leukemia, meaning a transformation to CBL from a pre-existing myeloid neoplasm $[3,4,7]$.

The World Health Organization has defined a classification of hematological malignancies, in which acute basophilic leukemia was included as a distinct entity but without any diagnostic criteria. Chronic basophilic leukemia was not included, and thus it is categorized as being part of the 'Myeloproliferative Neoplasms (MPN), unclassifiable' [8]. Subsequently, there are no universally accepted diagnostic criteria for CBL. There is a consensus between several research groups that the following characteristics should be present to diagnose CBL: persistent ( $>8$ weeks) hyperbasophilia (absolute count $\geq 1 \times 10^{3} / \mathrm{mm}^{3}$ ), relative count of basophils in bone marrow or peripheral blood of $\geq 40 \%$, with basophils being derived from a malignant clone, and lastly, the number of blast cells accounts for $<20 \%$ of all cells $[7,9]$. Possible additional diagnostic criteria are an increase in megakaryocytes, eosinophilic and basophilic hyperplasia resulting in a suppression of the neutrophilic lineage [7]. Our patient met all these criteria and, in the absence of any underlying myeloproliferative neoplasm, was diagnosed with primary chronic basophilic leukemia.

Various cytogenetic abnormalities have been described in patients with CBL, such as trisomy 8, del (11q), monosomy 7 [3], $t(5 ; 12)$ [4], and abnormal IL-6 (interleukine-6) gene expression in neoplastic basophils [5]. To date, no recurrent defect, either chromosomal or molecular, has been identified in CBL [9] and thus, there is no information on a possible driver mutation for the disease. As mentioned earlier, we performed NGS on the patient's bone marrow, which showed three mutations.

The likely pathogenic missense variant in the DNMT3A gene (P904A) has been reported in a few studies but this variant is rare, especially when compared to the well-known hotspot R882 [10]. DNMT3A mutations are most often seen as a CHIP-mutation (clonal hematopoiesis of indeterminate potential) which can be observed frequently in older patients without a hematological malignancy [11]. Subsequently, it could be possible this was not the driver mutation in this case. The pathogenic missense variant in the IDH2 gene belongs to the epigenetic modifiers, is known as a gain-of-function mutation, and is frequently described as a driver for acute myeloid leukemia [12]. The involvement of BCOR alterations in clonal disorders of myeloid lineage has been assessed in acute myeloid leukemia [13]. Both could be the driver mutation in this case of CBL. Because there are no NGS data available from patients with CBL thus far, it cannot be stated that one of these variants is in fact the driving genetic abnormality of CBL.

Treatment options for CBL remain limited. Two patients with primary CBL were treated with an allogeneic stem cell transplant [3]. In one of them this was successful, while the other died of procedurerelated complications soon after the transplantation [3]. In most patients the disease was palliated with hydroxyurea, with some surviving more than 48 months after diagnosis of CBL [7].

\section{Conclusion}

Chronic basophilic leukemia remains a poorly understood illness, as there are few reports of the disease. This report provides additional information on the clinical and laboratory findings related to the disease as well as novel insights in the underlying pathogenic mutations in several genes, namely the IDH2, DNMT3A, and BCOR gene. More research is needed to determine the specific characteristics and genetic makeup of chronic basophilic leukemia to be able to define it as a distinct entity different from other unclassifiable MPNs.

\section{Acknowledgements}

The authors owe special thanks to Brightcore for performing the Next Generation Sequencing. Additionally, we would like to thank Philippe Giron, Ken Maes and Freya Vaeyens for their help in interpreting the NGS findings.

\section{References}

1. Froyen G, Le Mercier M, Lierman E, Vandepoele K, Nollet F, Boone E, et al. Standardization of Somatic Variant Classifications in Solid and Haematological Tumours by a Two-Level Approach of Biological and Clinical Classes: An Initiative of the Belgian ComPerMed Expert Panel. Cancers (Basel). 2019; 11

2. Kyle RA, Pease GL. Basophilic leukemia. Arch Intern Med. 1966; 118: 205210.

3. Pardanani AD, Morice WG, Hoyer JD, Tefferi A. Chronic basophilic leukemia: a distinct clinico- pathologic entity? Eur J Haematol. 2003; 71: 18-22.

4. Tang G, Woods LJ, Wang SA, Brettler D, Andersen M, Miron PM, et al Chronic basophilic leukemia: a rare form of chronic myeloproliferative neoplasm. Hum Pathol. 2009; 40: 1194-1199.

5. Cehreli C, Ates H, Cehreli R, Sercan Z, Demirkan F. New paraneoplastic syndrome in chronic basophilic leukemia. Int J Hematol. 2013; 97: 498-504.

6. Cehreli C, Alacacioglu I, Piskin O, Ates H, Cehreli R, Calibasi G, et al. Mast cell leukemia associated with undefined morphology and chronic basophilic leukemia. BMC Hematol. 142014. p. 17

7. Çehreli C. Diagnostic Problems in Chronic Basophilic Leukemia. Turk J Haematol. 2018; 35: 283-289.

8. Arber DA, Orazi A, Hasserjian R, Thiele J, Borowitz MJ, Le Beau MM, et al The 2016 revision to the World Health Organization classification of myeloid neoplasms and acute leukemia. Blood. 2016; 127: 2391-2405

9. Valent P, Sotlar K, Blatt K, Hartmann K, Reiter A, Sadovnik I, et al. Proposed diagnostic criteria and classification of basophilic leukemias and related disorders. Leukemia. 2017; 31: 788-797.

10. Gaidzik VI, Schlenk RF, Paschka P, Stölzle A, Späth D, Kuendgen A, et al. Clinical impact of DNMT3A mutations in younger adult patients with acute myeloid leukemia: results of the AML Study Group (AMLSG). Blood. 2013; 121: 4769-4777.

11. Midic D, Rinke J, Perner F, Müller V, Hinze A, Pester F, et al. Prevalence and dynamics of clonal hematopoiesis caused by leukemia-associated mutations in elderly individuals without hematologic disorders. Leukemia. 2020; 34: 2198-2205.

12. DiNardo CD, Cortes JE. Mutations in AML: prognostic and therapeutic implications. Hematology Am Soc Hematol Educ Program. 2016; 2016: 348355.

13. Astolfi A, Fiore M, Melchionda F, Indio V, Bertuccio SN, Pession A. BCOR involvement in cancer. Epigenomics. 2019; 11: 835-855. 